

\title{
Electron-Hole Correlation in Fractional Quantum Hall Systems
}

\author{
P. Béran \\ Institut de Physique, Université de Neuchâtel, CH-2000 Neuchâtel, Switzerland
}

(Received)

\begin{abstract}
The 2D system of electron confined to the lowest Landau level is described using a representation of the density matrix depending both on electron and hole coordinates. Condensation of the electron system into a fractional quantum Hall state is associated to clustering of particle and hole coordinates. The correlation of particle and hole coordinates is studied and groundstate wave functions are derived for $\nu=p /(2 p \pm 1)$. These wave functions prove to be accurate for the studied cases $\nu=2 / 3$ and $3 / 5$ and are identical to pair wave functions [R. Morf et al., Phys. Rev. B34, 3037 (1986)] for $\nu=2 / 3$ and $2 / 5$.

PACS numbers: $73.40 . \mathrm{Hm}$
\end{abstract}

The theory of Laughlint provides a good microscopic description of the $\nu=1 / m$ incompressible quantum Hall states, with $m$ odd. Based on this description, it has been proposed to characterize fractional quantum Hall states by the occurence of binding of flux quanta to electronst. An explicit formulation for such a binding is provided by the composite Fermion approach 3 , which makes use of a singular gauge transformation to map the system of electrons in presence of magnetic field onto a system of composite Fermions, which are objects composed of an electron bound to an even number of flux quanta.

In this note, an alternative formulation is presented for treating the binding of flux quanta to electrons. This approach makes use of a particular representation of the finite temperature density matrix for the electron system confined to the lowest Landau level which consists in matrix elements of the density matrix operator evaluated between states parametrized by electron coordinates and states parametrized by the coordinates of electron vacancies, i.e. holes. The latter coordinates can be regarded as positions of flux quanta.

This formulation, in which electron-hole symmetry is fully explicit, leads to a simple and natural picture for the incompressible quantum Hall state at $\nu=p / q$ : At some finite temperature, the density matrix is large only for configurations in which electron and hole coordinates can be grouped into clusters containing each $p$ electron coordinates and $q-p$ hole coordinates. This electronhole binding results dynamically from the repulsion between electrons. The creation of a neutral excitation corresponds to the breaking of such a cluster into two smaller clusters, each one leading to a local fractional charge defect. The fact that this breaking requires a finite amount of potential energy leads to a gap in the energy spectrum and to incompressibility. The off-diagonal long range order characterizing incompressible groundstates 5 results from the fact that these clusters are Bosons and Bosecondense.

The advantage of working with this finite temperature description is that the correlation between hole coordinates, which play the role of positions of flux quanta, and electron coordinates can be written down explicitely. However, the price to pay is the work necessary to derive from this density matrix a microscopic description of the system a zero temperature. We show how to obtain groundstate wave functions either as a function of electron coordinates or as a function of hole coordinates in the case of the fractional quantum Hall states at $\nu=p /(2 p+1)$. The case is made that the wavefunctions derived from this density matrix are generalizations of the pair wavefunctions of Ref.6, which are known to be accurate. In the latter wave functions, the pairing of electrons can be regarded as resulting directly from the presence in the density matrix of clusters containing each $p=2$ electrons and $q-p$ holes.

This description of fractional quantum Hall states based on a density matrix is in fact analogous the one provided by the composite Fermion approach 3 - 4 . Indeed, a cluster made up of one electron and one hole corresponds to a composite Fermion and a cluster made up of $p$ electrons and $p+1$ holes, which enters into the description of the $\nu=p /(2 p+1)$ state, corresponds to $p$ composite Fermions, each one belonging to a different Landau level, all bound to a flux quantum of the field experienced by composite Fermions.

Let us first study the density matrix of the 2D electron gas confined to the lowest Landau level at a filling factor $\nu=p / q$ characterized by the presence of an incompressible groundstate. More specifically, we consider the quantity

$$
\rho_{\beta}\left(w_{1} \ldots w_{h N} z_{1} \ldots z_{p N}\right)=\left\langle w_{1} \ldots w_{h N}\left|e^{-\beta \tilde{V}}\right| z_{1} \ldots z_{p N}\right\rangle
$$

where $\tilde{V}$ is the electron-electron interaction projected onto the lowest Landau level and where $\left|z_{1} \ldots z_{p N}\right\rangle$ and $\left|w_{1} \ldots w_{h N}\right\rangle$ are states respectively given in electron representation and hole representation, defined by

$$
\begin{aligned}
\left|z_{1} \ldots z_{p N}\right\rangle & =c^{\dagger}\left(z_{1}\right) \ldots c^{\dagger}\left(z_{p N}\right)|0\rangle, \\
\left|w_{1} \ldots w_{h N}\right\rangle & =c\left(w_{1}\right) \ldots c\left(w_{h N}\right)|1\rangle
\end{aligned}
$$

where $|0\rangle$ and $|1\rangle$ respectively denote the empty- and fullLandau level states, $c^{\dagger}(z)$ is the operator creating an elec- 
tron in a coherent state centered at position $z, h$ is given by $h=q-p$ and where $p N$ and $h N$ are respectively the number of electrons and holes in the lowest Landau level.

For $\beta=0$, the density matrix of Eq. (11) is simply given by

$$
\begin{gathered}
\rho_{0}=\prod_{j_{1}<j_{2}}^{h N}\left(w_{j_{1}}-w_{j_{2}}\right) \prod_{i_{1}<i_{2}}^{p N}\left(z_{i_{1}}-z_{i_{2}}\right) \\
\times \prod_{j=1}^{h N} \prod_{i=1}^{p N}\left(z_{i}-w_{j}\right) \prod_{j=1}^{h N} e^{-\frac{\left|w_{j}\right|^{2}}{4}} \prod_{i=1}^{p N} e^{-\frac{\left|z_{i}\right|^{2}}{4}}
\end{gathered}
$$

in units of the magnetic length, where $z_{i}$ and $w_{j}$ are respectively the complex coordinates of electron $i$ and hole $j$. Ignoring for a moment the distinction between variables $z_{i}$ and $w_{j}$, we see that $\rho_{0}$ has a functional form identical to that of the wave function describing a full Landau level. Thus configurations $\left(w_{1} \ldots w_{h N} z_{1} \ldots z_{p N}\right)$ characterized by non-negligeable values of $\rho_{0}$ have for feature ( denoted by (A) ) that their coordinates are homogeneously distributed over the whole sample. Let us now study the effect of the electron-electron repulsion present in Eq. (11). In the case of a repulsion characterized by vanishing pseudo-potential coefficients for even values of the electron-electron relative angular momentum, the Hamiltonian matrix is given, up to an additive constant, by

$$
\begin{array}{r}
-\frac{\partial}{\partial \beta} \rho_{\mid \beta=0}\left(w_{1} \ldots w_{h N} z_{1} \ldots z_{p N}\right)=-\sum_{i=1}^{p N} \sum_{j=1}^{h N} \sum_{L} \tilde{V}_{L} \\
\times \int d^{2} \xi\left[\mathcal{P}_{\psi_{L}^{(\xi)}\left(z_{i} w_{j}\right)} \rho_{0}\right]\left(w_{1} \ldots w_{h N} z_{1} \ldots z_{p N}\right),
\end{array}
$$

where the sum over $L$ is carried over odd positive integers, $\tilde{V}_{L}$ denote the pseudo-potential coefficients, $\psi_{L}^{(\xi)}\left(z_{1} z_{2}\right)$ is the wave function describing two particles with relative angular momentum $L$ and with center of mass in a coherent state centered at position $\xi$ and where $\mathcal{P}$ is the projector associated to this wave function, defined by

$$
\begin{aligned}
& {\left[\mathcal{P}_{\psi_{L}^{(\xi)}\left(z_{i} w_{j}\right)} \rho_{0}\right]\left(w_{1} \ldots w_{h N} z_{1} \ldots z_{p N}\right)=\psi_{L}^{(\xi)}\left(z_{i} w_{j}\right) \times} \\
& \int d^{2} z_{i}^{\prime} d^{2} w_{j}^{\prime} \psi_{L}^{(\xi)^{*}}\left(z_{i}^{\prime} w_{j}^{\prime}\right) \rho_{0}\left(w_{1} . . w_{j}^{\prime} . . w_{h N} z_{1} . . z_{i}^{\prime} . . z_{p N}\right) .
\end{aligned}
$$

As can be seen in Eq. (5), the effect of the interaction at finite values of $\beta$ is to increase $\rho_{\beta}$ for configurations which have for feature (denoted by (B)) that their coordinates $w_{j}$ are close to their coordinates $z_{i}$. Thus it is likely that the quantity $\rho_{\beta}$ for small but finite $\beta$ will be largest for configurations $\left(w_{1} \ldots w_{h N} z_{1} \ldots z_{p N}\right)$ in the $2 \mathrm{D}$ plane which can be divided into compact clusters of equal sizes containing each $p$ positions $z_{i}$ and $h$ positions $w_{j}$, which possess both features (A) and (B).

Let us now construct a form for the density matrix satisfying the latter requierement for the special case of the filling fractions $\nu=p /(2 p+1)$. The question of the temperature range in which this form is valid will be discussed latter. We propose to use the function given by $\kappa\left(w_{1} \ldots w_{h N} z_{1} \ldots z_{p N}\right)=\mathcal{A}_{w} \mathcal{A}_{z} \hat{\kappa}\left(w_{1} \ldots w_{h N} z_{1} \ldots z_{p N}\right)$ and by

$\hat{\kappa}=\int \prod_{n=1}^{N}\left[d^{2} \xi_{n} \mathcal{P}_{\chi^{(\xi n)}\left(z_{p(n-1)+1} \ldots z_{p n} w_{h(n-1)+1} \ldots w_{h n}\right)}\right] \rho_{0}$,

where $\mathcal{A}_{w}$ and $\mathcal{A}_{z}$ are antisymmetrization operators acting respectively on sets of variables $\left(w_{1} \ldots w_{h N}\right)$ and $\left(z_{1} \ldots z_{p N}\right)$ and where $\chi^{\left(\xi_{n}\right)}$ is a function describing the correlation of $p$ electrons and $h=p+1$ holes in a cluster centered at position $\xi_{n}$, given by

$$
\begin{aligned}
\chi^{(\xi)}\left(z_{1} . . z_{p} w_{1} . . w_{h}\right) & =\prod_{i_{1}<i_{2}}^{p}\left(z_{i_{1}}-z_{i_{2}}\right) \prod_{j_{1}<j_{2}}^{h}\left(w_{j_{1}}-w_{j_{2}}\right)^{3} \\
\times & \prod_{i=1}^{p} e^{\frac{\xi^{*} z_{i}}{2}-\frac{\left|z_{i}\right|^{2}+|\xi|^{2}}{4}} \prod_{j=1}^{h} e^{\frac{\xi^{*} w_{j}}{2}-\frac{\left|w_{j}\right|^{2}+|\xi|^{2}}{4}} .
\end{aligned}
$$

The projectors and the term $\rho_{0}$ in Eq. (7) respectively ensure that configurations leading to a large value of $\kappa$ have features (B) and (A). The integrals implicit in the projectors of Eq. (7) can be evaluated using the identity

$$
\begin{aligned}
& \int \prod_{i=1}^{n}\left[d^{2} z_{i} e^{-\frac{\left|z_{i}\right|^{2}+\xi z_{i}^{*}}{2}}\right] P_{k}^{*}\left(z_{1} . . z_{n}\right) Q_{l}\left(z_{1} . . z_{n}\right) f\left(z_{1} . . z_{n}\right)= \\
& f(\xi . . \xi) \int \prod_{i=1}^{n}\left[d^{2} z_{i} e^{-\frac{\left|z_{i}\right|^{2}}{2}}\right] P_{k}^{*}\left(z_{1} . . z_{n}\right) Q_{l}\left(z_{1} . . z_{n}\right),
\end{aligned}
$$

where $f$ is a polynomial and where $P_{k}$ and $Q_{l}$ are homogeneous polynomials of total degree $k$ and $l$ with $k \leq l$ and are invariant under global translations of their variables 9 . This leads, up to a multiplicative constant, to

$$
\begin{aligned}
& \hat{\kappa}=\int \prod_{n=1}^{N}\left[d^{2} \xi_{n} \chi^{\left(\xi_{n}\right)}\left(z_{p(n-1)+1} \ldots z_{p n} w_{h(n-1)+1} \ldots w_{h n}\right)\right] \\
& \times \prod_{n_{1}<n_{2}}^{N}\left(\xi_{n_{1}}-\xi_{n_{2}}\right)^{q^{2}} \prod_{n=1}^{N} e^{-\frac{q\left|\xi_{n}\right|^{2}}{4}} .
\end{aligned}
$$

The function $\chi$ is choosen so as to satisfy the following criteria: (a) $\chi^{(\xi)}$ is antisymmetric under interchange of two electron coordinates or under interchange of two hole coordinates. (b) The total degree of the polynomial part of $\chi^{(0)}$ equals $q(q-1) / 2$. Indeed, in light of Eq. (9), $\kappa$ would vanish for a smaller degree. On the other hand, a larger degree would lead 9 to a reduction of the power of factors $\left(\xi_{n_{1}}-\xi_{n_{2}}\right)$ in Eq. (10) and configurations with a large value of $\kappa$ would not satisfy condition (A) anymore. (c) Factors of type $\left(z_{i}-w_{j}\right)$ are absent so that $\chi$ is large for configurations satisfying condition (B).

In order to support our claim that the function $\kappa$ provides a realistic description of the density matrix for some temperature, we now make the case that the groundstate wave functions derived from this form for the density matrix are generalizations of the pair wave functions of 
Refl, which are known to be accurate. Let us denote by $\Phi\left(z_{1} \ldots z_{p N}\right)$ and $\Theta\left(w_{1} \ldots w_{h N}\right)$ the wave functions describing the groundstate at $\nu=p /(2 p+1)$ in the electron- and hole-representations of Eqs. (2) and (3), respectively. If $\kappa$ provides a good description of the density matrix, then $\Phi$ and $\Theta$ should maximize the quantity

$$
R(\Phi, \Theta)=\frac{\int d^{2 p N} Z d^{2 h N} W \kappa^{*}(W, Z) \Phi(Z) \Theta(W)}{\sqrt{\int d^{2 p N} Z|\Phi(Z)|^{2} \int d^{2 h N} W|\Theta(W)|^{2}}}
$$

where $Z \equiv\left(z_{1} \ldots z_{p N}\right)$ and $W \equiv\left(w_{1} \ldots w_{h N}\right)$.

We now approximate $R(\Phi, \Theta)$ by substituting $\hat{\kappa}$ for $\kappa$ in Eq. (11). Ignoring for the moment the constraint of antisymmetrization, we consider wave functions of the type

$$
\begin{aligned}
& \hat{\Phi}\left(z_{1} \ldots z_{p N}\right)=\prod_{i=1}^{p N} e^{-\frac{\left|z_{i}\right|^{2}}{4}} \prod_{n=1}^{N} a_{l_{a}}\left(z_{(n-1) p+1} \ldots z_{n p}\right) \\
& \quad \times \prod_{n_{1}<n_{2}}^{N} b_{l_{b}}\left(z_{\left(n_{1}-1\right) p+1} \ldots z_{n_{1} p}, z_{\left(n_{2}-1\right) p+1} \ldots z_{n_{2} p}\right)
\end{aligned}
$$

where $a_{l_{a}}$ and $b_{l_{b}}$ are translationally invariant and homogeneous polynomials of total degree $l_{a}$ and $l_{b}$ to be determined. The polynomial $a_{l_{a}}\left(z_{(n-1) p+1} \ldots z_{n p}\right)$ describes correlations within the group $n$ of variables $z_{(n-1) p+1} \ldots z_{n p}$. The polynomial $b_{l_{b}}\left(z_{\left(n_{1}-1\right) p+1} \ldots z_{n_{1} p}, z_{\left(n_{2}-1\right) p+1} \ldots z_{n_{2} p}\right)$ is built using products of $l_{b}$ factors of type $\left(z_{\left(n_{1}-1\right) p+i_{1}}-z_{\left(n_{2}-1\right) p+i_{2}}\right)$, with $i_{1}, i_{2}=1 \ldots p$, and describes correlations between groups $n_{1}$ and $n_{2}$. We write similarly

$$
\begin{aligned}
& \hat{\Theta}\left(w_{1} \ldots w_{h N}\right)=\prod_{j=1}^{h N} e^{-\frac{\left|w_{j}\right|^{2}}{4}} \prod_{n=1}^{N} c_{l_{c}}\left(w_{(n-1) h+1} \ldots w_{n h}\right) \\
& \quad \times \prod_{n_{1}<n_{2}}^{N} d_{l_{d}}\left(w_{\left(n_{1}-1\right) h+1} \ldots w_{n_{1} h}, w_{\left(n_{2}-1\right) h+1} \ldots w_{n_{2} h}\right) .
\end{aligned}
$$

We now introduce Eqs. (10), (12), and (13) into Eq. (11) and integrate over coordinates $z_{i}$ and $w_{j}$ before $\xi_{n}$ in the numerator. In order to maximize the numerator, we demand that the result of the integration over electron and hole coordinates have the same dependence in variables $\xi_{n}$ as the kernel of $\hat{\kappa}$ given by the second linf of Eq. (10). Using Eq. (9), it can be seen by inspection 9 that this is possible only when $l_{a}$ and $l_{c}$ are equal to the degrees of polynomial parts of Eq. (\$) depending respectively on coordinates $z_{i}$ and $w_{j}$, i.e. $l_{a}=p(p-1) / 2$ and $l_{c}=3 h(h-1) / 2$, and when $l_{b}+l_{d}=q^{2}$. The latter condition, together with the constraint that each coordinate in $\hat{\Phi}$ or in $\hat{\Theta}$ have $q N-1$ zeros, leads to $l_{b}=p q$ and $l_{d}=h q$. In light of Eqs. (9) and (8), it is judicious to choose

$$
a_{l_{a}}\left(z_{1} \ldots z_{p}\right)=\prod_{i_{1}<i_{2}}^{p}\left(z_{i_{1}}-z_{i_{2}}\right)
$$

$$
c_{l_{c}}\left(w_{1} \ldots w_{h}\right)=\prod_{j_{1}<j_{2}}^{h}\left(w_{j_{1}}-w_{j_{2}}\right)^{3}
$$

in order to obtain a large result from integration over particle and hole coordinates in the numerator of Eq. (11). Among polynomials $b_{p q}$ and $d_{h q}$ leading to the same value for the numerator, those yielding to the most homogeneous distribution of factors $\left(z_{i_{1}}-z_{i_{2}}\right)$ over pairs $1 \leq i_{1}<i_{2} \leq p N$ and of factors $\left(w_{j_{1}}-w_{j_{2}}\right)$ over pairs $1 \leq j_{1}<j_{2} \leq h N$ lead to the smallest denominator. We therefore choose

$$
\begin{aligned}
b_{p q}\left(z_{1} \ldots z_{2 p}\right)= & \prod_{i_{1}=1}^{p} \prod_{i_{2}=1}^{p}\left(z_{i_{1}}-z_{p+i_{2}}\right)^{2} \\
& \times \sum_{P_{p}} \prod_{i=1}^{p}\left(z_{P_{i}}-z_{p+i}\right)
\end{aligned}
$$

and

$$
\begin{aligned}
d_{h q}\left(w_{1} \ldots w_{2 h}\right)= & \prod_{j_{1}=1}^{h} \prod_{j_{2}=1}^{h}\left(w_{j_{1}}-w_{h+j_{2}}\right)^{2} \\
& \times \sum_{P_{h}} \prod_{j=1}^{h}\left(w_{P_{j}}-w_{h+j}\right)^{-1}
\end{aligned}
$$

where $P_{p}$ and $P_{h}$ respectively denote sums over permutations of $p$ and $h$ objects.

The wave functions $\Phi=\mathcal{A}_{z} \hat{\Phi}$ and $\Theta=\mathcal{A}_{w} \hat{\Theta}$ defined using Eqs. (12), (13), (14), (15), (16) apd (17) are identical to the pair wave functions of Ref. 6 when $p=2$ and $h=2$, respectively. $\Phi$ and $\Theta$ provide a good microscopic description of groundstates at filling factors $\nu=p /(2 p+1)$ and $\nu=h /(2 h-1)$, respectively. Indeed, they lead to numbers of flux quanta which are in agreement with the prescription of the hierarchical scheme 8 and have a large overlap with the exact groundstates of the corresponding systems of particles on a sphere in presence of Coulomb repulsion: In the case $\nu=2 / 3$ and for 8 and 10 particles respectively, the overlaps are 0.954 and 0.930 whereas the numbers of $(L=0)$ states are 2 and 6 . In the case $\nu=3 / 5$ and for 6 and 9 particles respectively, the overlaps are 0.988 and 0.970 and the numbers of $(L=0)$ states are 3 and 8 .

We now study the constraints imposed by statistics on allowed values of parameters $p, h$ and $q=p+h$ using a path integral representation of the partition function

$$
\mathcal{Z}(\beta)=\int d^{2} z_{1} \ldots d^{2} z_{p N}\left\langle z_{1} \ldots z_{p N}\left|e^{-\beta \tilde{V}}\right| z_{1} \ldots z_{p N}\right\rangle .
$$

The exponential in Eq. (18) is brocken into $M$ pieces $\exp (-\beta \tilde{V} / M)$ with $M$ even, and projectors $\left|z_{1}^{(m)} \ldots z_{p N}^{(m)}><z_{1}^{(m)} \ldots z_{p N}^{(m)}\right|$ and $\mid w_{1}^{(m)} \ldots w_{h N}^{(m)} \quad><$ $w_{1}^{(m)} \ldots w_{h N}^{(m)} \mid$ are inserted alternatively at imaginary times $m \beta / M$ for $m$ even and $m$ odd, respectively. We consider configurations which can be divided at all imaginary times in clusters containing $p$ coordinates $z_{i}^{(m)}$ and 
$h$ coordinates $w_{j}^{(m)}$, which significantly contribute to the partition function. For simplicity we further demand that $z_{i}^{(m)}$ and $w_{j}^{(m)}$ have a smooth dependence on $m$ and belong to the same cluster throughout imagimary time. The phase corresponding to a given path is a product of phase contributions associated to each time step, which can in turn be evaluated by means of Eq. (1). Ignoring factors $\left(z_{i}-w_{j}\right)$ for $z_{i}$ and $w_{j}$ belonging to the same cluster, this leads to $(-1)^{p h[P]}$, where $[P]$ is the parity of the permutation taking place among clusters between imaginary time $\tau=0$ and $\tau=\beta$. Thus such paths contribute constructively to the partition function only if $p h$ is even, that is if (a) $p$ is even and $h$ is odd or (b) $p$ is odd and $h$ is even or (c) both $p$ and $h$ are even. Note that cases (a) and (b) imply a filling factor $\nu=p / q$ with odd denominator.

The fact that all incompressible fractional quantum Hall states observed in the lowest Landau level are characterized by a filling factor with odd denominator may be attributed to the fact that a stable cluster made up of $p$ electron coordinates and $h$ hole coordinates can only be obtained if one of the parameters is odd, thus corresponding to case (a) or (b). Indeed, for $p$ and $q$ both even, the paths obtained by clustering $p$ coordinates $z_{i}$ and $h$ coordinates $w_{j}$ could be energetically unfavorable compared with paths obtained by clustering $p / 2$ coordinates $z_{i}$ and $h / 2$ coordinates $w_{j}$.

The incompressible state at $\nu=5 / 2$ is characterized by $p=h=2$ ( case (c)). The absence of incompressible state at $\nu=1 / 2$ is attributed 10 to the fact that, in the lowest Landau level, clusters with $p=h=2$ are unstable toward the formation of clusters with $p=h=1$. Since the paths of such $p=h=1$ clusters contribute to the partition function with a phase $(-1)^{[P]}$ reminiscent $f^{f}$ Fermions, they can be regarded as composite Fermionst.

It is important to note that the approximation to the density matrix provided by function $\kappa$ of Eq. (10) is unable to describe neutral excitations consisting in pairs of well-separated fractionally-charged excitations. Indeed, when using this approximate form of the density matrix to evaluate the number of electrons in a surface large compared to the squared magnetic length, one obtains fluctuations much too small to include the effect of such excitations. In order to include the effect of a single quasiparticle-quasihole pair, it is necessary to add to $\kappa$ a further term. This additional term can be obtained from the right-hand side of Eq. (7) by replacing one of the projectors associated to clusters of $p$ electrons and $h$ holes by two projectors: One associated to a cluster of $p^{\prime}$ electrons and $h^{\prime}$ holes and an other associated to a cluster of $p-p^{\prime}$ electrons and $h-h^{\prime}$ holes, with $p^{\prime}$ and $h^{\prime}$ satisfying $p^{\prime} h-p h^{\prime}= \pm 1$. The cluster associated to the plus sign leads to a local charge defect $e / q$ (quasiparticle) and the other to $-e / q$ (quasihole), where $e$ is the electron's charge. The parameters $p^{\prime}$ and $h^{\prime}$ are given by $\left.\left.p^{\prime} / h^{\prime}=1 /\left(n_{1}+1 /\left(n_{2}+\ldots+1 / n_{k-1}\right)\right) \ldots\right)\right)$ in terms of the continued fraction decomposition $p / h=$ $\left.\left.1 /\left(n_{1}+1 /\left(n_{2}+\ldots+1 / n_{k}\right)\right) \ldots\right)\right)$. Thus the creation of a neutral excitation corresponds to the breaking of a cluster of $p$ electrons and $h$ holes. The fact that this breaking requires a finite amount of potential energy, together with the fact that $\kappa$ of Eq. (77) considered as a function of $z_{1} \ldots z_{p N}$ or of $w_{1} \ldots w_{h N}$ has a small overlap with the contribution described above including a neutral excitation, leads to a gap in the energy spectrum and to incompressibility.

We finally address the question of the temperature range in which the function $\kappa\left(w_{1} \ldots w_{h N} z_{1} \ldots z_{p N}\right)$ of $\mathrm{Eq}$ .(7) may be adequate to describe the density matrix of Eq. (11). Although $\kappa$ is unable to describe neutral excitations consisting in pairs of well-separated elementary excitations which are associated to short wavelength collective modes 11 , We do believe that the effect of long wavelength collective modes are included in $\kappa\left(w_{1} \ldots w_{h N} z_{1} \ldots z_{p N}\right)$ and are responsible for the correlations existing between variables $z_{i}$ and $w_{j}$. This collective modes are characterized by a finite excitation energy $\epsilon_{k=0}$. We thus conjecture that the temperature for which $\kappa$ is adequate to describe the density matrix is of the order of $\epsilon_{k=0} / k_{B}$.

\section{ACKNOWLEDGEMENTS}

We would like to thank R. Morf for helpfull discussions. We acknowledge support from the Swiss National Science Fundation under grand No. 2000-040395.94/1.

${ }^{1}$ R.B. Laughlin, Phys. Rev. Lett. 50, 1395 (1983).

${ }^{2}$ N. Read, Phys. Rev. Lett. 62, 86 (1989).

${ }^{3}$ J.K. Jain, Phys. Rev. Lett. 63, 1223 (1989).

${ }^{4}$ B.I. Halperin, P.A. Lee and N. Read, Phys. Rev. B47, 7312 (1993).

${ }^{5}$ S.M. Girvin and A.H. MacDonald, Phys. Rev. Lett. 58, 1252 (1987).

${ }^{6}$ R. Morf, N. d'Ambrumenil and B.I. Halperin, Phys. Rev. B34, 3037 (1986).

${ }^{7}$ S.M. Girvin and T. Jach, Phys. Rev. B29, 5617 (1984).

${ }^{8}$ F.D.M. Haldane in The Quantum Hall Effect, ed. R.E. Prange and S.M. Girvin, Springer 1987.

${ }^{9}$ This identity can be established using a transformation to center of mass and relative coordinates. If $k>l$, the lefthand side of Eq. (9) is proportional to a linear combination of terms of the type $\partial_{z_{1}}^{l_{1}} \ldots \partial_{z_{n}}^{l_{n}} f_{\mid z_{1}=\ldots=z_{n}=\xi}$ with $l_{1}+\ldots+l_{n}=$ $k-l$.

10 T.L. Ho, Phys. Rev. Lett. 75, 1186 (1995).

${ }^{11}$ R.B. Laughlin in The Quantum Hall Effect, ed. R.E. Prange and S.M. Girvin, Springer 1987. 